\title{
Long-term Polarimetric Activity of the Cool Supergiant $\mu$ Cephei
}

\author{
J. Arsenijević and S. Jankov \\ Astronomical Observatory, Beograd, Yugoslavia
}

\begin{abstract}
The intrinsic polarisation parameters $P, \theta, Q$ and $U$ of the star $\mu$ Cep, in the $V$ spectral region during the period 1979-1989, are presented. The data were obtained from the Belgrade polarimetric observational program. The polarization percentage of the star exhibits change is of about $2.5 \%$ during this interval and the position angles change by about 90 degrees. These very large long-term changes of polarization parameters are discussed briefly.
\end{abstract}

\section{Introduction and observations}

The cool bright supergiant $\mu$ Cep (HD 206936) has been studied polarimetrically for a long period of time by many authors. The first measurement was made by Hiltner (1951). Grigoryan (1959) discovered the variability of its polarization. The scattering by grains in a circumstellar envelope was adopted as a mechanism to produce polarization. The empirical schematic model proposed by Polyakova (1984), for example, was used to explain the intrinsic polarization and brightness variation of $\mu$ Cep by the rotation modulation and intrinsic variation of two active antipodal spot regions. The polarization was supposed to be a product of starlight scattered by dust streaming from active regions. This problem is far from solution.

Polarimetric observations presented in this paper were carried out with the $65 \mathrm{~cm}$ Zeiss refractor and the Belgrade stellar polarimeter (Kubičela et al., 1976) in the $V$ spectral region during the period 1979-1989. The polarimeter was modified in 1979 to digitalize and record the data. The angular velocity of the analyser was one turn per minute. In most cases "one measurement" (one point in the Figs. 1-3) can represent up to 8-polarimetric phase-averaged 1-minute sine-wave signals. The typical standard deviation of the observed Stokes parameters for one 8-minute measurement of this star is not higher than $0.07 \%$. During the whole interval of observation the instrumental system was carefully checked by measuring 11 polarized standard stars and 17 stars known to have zero polarization. For the interstellar polarization parameters in the direction of $\mu$ Cep the estimated values of Polyakova (1974) $(Q=-0.1 \%, U=1.5 \%)$ were used as the most reliable among 
those published. The individual values of the intrinsic polarization percentage $P$, the position angle $\theta$ and the corresponding Stokes parameters $Q$ and $U$ in the period 1979-1989 are presented in Figs. 1-3.

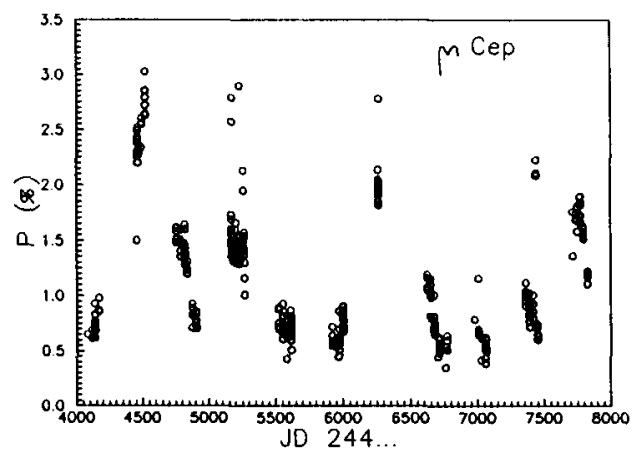

Fig. 1. The intrinsic polarization percentage $P$.

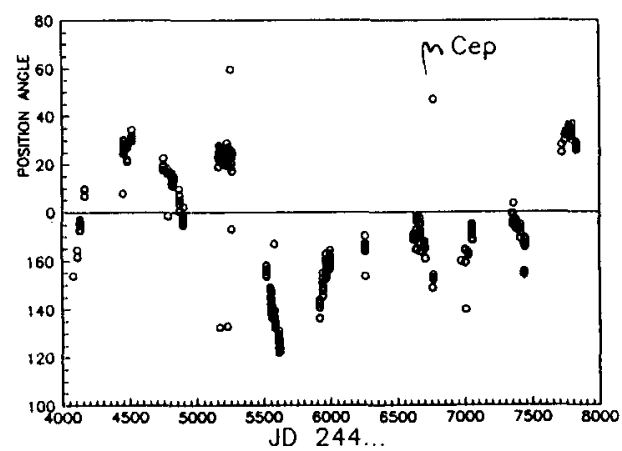

Fig. 2. The intrinsic polarization angle $\theta$.

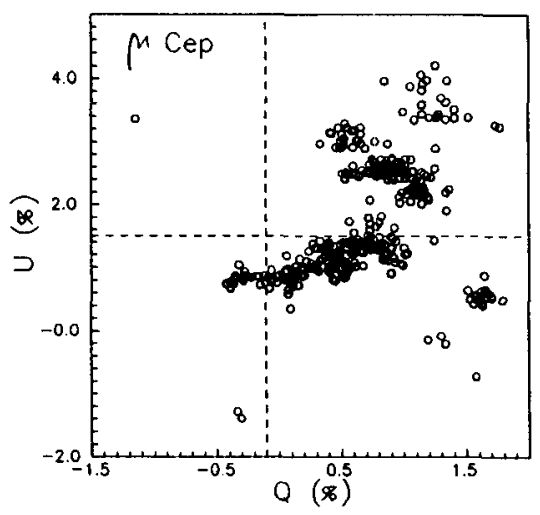

Fig. 3. The stokes parameters $Q$ and $U$. The interstellar polarization is given by the intersection of the dashed lines representing also the intrinsic polarization coordinate system.

The observed and intrinsic Stokes parameters are not presented separately because the relative position of these parameters do not change after extracting the interstellar component. So, only the origin of the coordinate system changes to the values $Q=-0.1 \%, U=1.5 \%$. In Fig. 3 the new coordinate axes (for the intrinsic polarization) are shown by the dashed lines. From Figs. 1-3 it is obvious that the polarization of $\mu$ Cep exhibit large changes. The intrinsic polarization percentage changes between values of about $0.35 \%$ and $3 \%$. The variation in the position angle are of the order of 90 degrees. 


\section{Discussion}

In the last several years the behaviour of the star has been extraordinary: high optical polarization during 1980 (Arsenijević et al., 1980) and a possible eruptive event on August 3, 1981 (Arsenijević, 1985) were observed. In addition, some successful attempts to see the envelope structure were made. The first image of the circumstellar envelope in the $\mathrm{Na}$ I $589 \mathrm{~nm}$ emission line was obtained on 1-2 September 1984 by Mauron et al. (1986) and, at last, observations of dust envelope polarization were made on June 1985 and June-July 1986 by Borgne and Mauron (1989).

We shall mention now only some interesting facts seen at first sight. There is an agreement between the polarization position angle of the envelope itself measured by Borgne and Mauron (1989), covering the very disk of the star, and the intrinsic polarization position angle presented here for the corresponding period of time in 1985 and 1986. Namely, for the region of high envelope polarization (in $V$ spectral region) in Borgne and Mauron, Table 2, the position angle of polarization is between 164 and 169 degrees while the intrinsic polarization position angle measured by us in the same period of time is almost in the same interval.

As global polarimetric parameters include the whole envelope, particularly the parts nearer to the star which are more efficient in producing polarized light, as well as the outer parts of the envelope having the angles of polarization mentioned, we can conclude that the geometry of the inner part of the star envelope was, at least during the mentioned period, similar to that of the outer one. The image of the circumstellar envelope in the $\mathrm{Na} \mathrm{I}$ emission line was observed (Mauron et al., 1986) in the interval of time when the envelope activity of the star, according to polarimetric observations, was small. Hence, one can perhaps conclude that the polarizing contribution of the gas component to the intrinsic polarization is also small.

Further discussion of some other physical parameters or processes (magnitude, flaring, spectral changes etc.) and their correlations with polarization parameters during the whole interval of observations will be presented later.

\section{References}

Arsenijević, J.: 1985, Astron. Astrophys. 145, 430

Arsenijević, J., Kubičela, A., Vince, I.: 1980, Inf. Bull. Var. Stars No 1859

Borgne Le, J.F., Mauron, N.: 1989, Astron. Astrophys. 210, 198

Grigoryan, K.A.: 1959, Soobshch. Byurakan. Obs. 27, 55

Hiltner,W.A.: 1951, Astrophys. J. 114, 241

Kubičela, A., Arsenijević, J., Vince, I.: 1976, Publ. Dept. Astron. Beograd 6, 25

Mauron, N., Cailloux, M., Tilloles, P., Le Fevre, O.: 1986, Astron. Astrophys. Letters 165, L9

Polyakova, T.A.: 1974, Astrofizika 10, 1

Polyakova, T.A.: 1984, Astrofizika 21, 125 